\title{
Physical Activity and Neural Correlates of Sad Facial Expressions in Premenstrual Syndrome
}

\author{
Ren-Jen Hwang ${ }^{1,2,{ }^{*} \text {, Hsin-Ju Chen }}{ }^{1}$, Zhan-Xian Guo ${ }^{1}$, Yu-Sheun Lee ${ }^{1,3}$, Yueh-O Chuang ${ }^{2}$ \\ ${ }^{1}$ Department of Nursing, Chang Gung University of Science and Technology, Taoyuan, Taiwan \\ ${ }^{2}$ Department of Nursing, Chang Gung Memorial Hospital, Taoyuan, Taiwan \\ ${ }^{3}$ Department of Nursing, China Medical University Hospital, Taichung, Taiwan
}

Email address:

rjhuang@mail.cgust.edu.tw (Ren-Jen Hwang), hjchen01@hotmail.com (Hsin-Ju Chen), critisec@gmail.com (Zhan-Xian Guo), q0o60824@yahoo.com.tw (Yu-Sheun Lee),j22092@cgmh.org.tw (Yueh-O Chuang)

${ }^{*}$ Corresponding author

\section{To cite this article:}

Ren-Jen Hwang, Hsin-Ju Chen, Zhan-Xian Guo, Yu-Sheun Lee, Yueh-O Chuang. Physical Activity and Neural Correlates of Sad Facial Expressions in Premenstrual Syndrome. Journal of Gynecology and Obstetrics. Vol. 6, No. 3, 2018, pp. 56-66. doi: $10.11648 /$ j.jgo.20180603.14

Received: May 1, 2018; Accepted: June 4, 2018; Published: June 28, 2018

\begin{abstract}
Exercise benefits our emotional function, particularly frontal lobe-mediated cognitive processes. Recent studies have indicated that affective neural circuits are modulated by premenstrual syndrome (PMS). Meanwhile, observational studies have reported that physical activity can promote PMS remission. Nonetheless, few studies have investigated the effect of physical activity on sad emotion recognition from a neurobiological perspective. Our objective was to explore the relationships between exercise and high order sad emotion cognitive processing in women with PMS. We conducted a sad facial emotion recognition task to examine the effects of aerobic exercise on activity in central frontal regions with electroencephalography in 31 participants. Participants were divided into a high (HPMS) or low severity PMS (LPMS) group according to self-reported PMS scores. We then analyzed cortical activity in response to sad cues, comparing such activity between the groups. Repeated ANOVA and pooled $t$ statistics were used for statistical analysis. We observed a significant reduction in the N250 wave evoked by sad emotions after exercise compared with baseline at most channels in HPMS $(\mathrm{P}<0.05)$, but not in LPMS. There was also a significant post-exercise prolongation of N250 latency at F3 and C3 in HPMS. There were no differences in N250 activation between the groups at baseline but significantly lower activation was noted in the lateral prefrontal cortex (PFC: F7 and F8) of HPMS compared with LPMS after exercise. Furthermore, higher PMS scores were significantly associated with greater PFCN250 amplitude before exercise, but this was not observed after exercise. Acute exercise induced significant brain activity changes in response to sad cues in the HPMS group only. Furthermore, significantly lower activation of the lateral PFC was found after exercise in HPMS compared with LPMS. Given our results, we discuss the potential efficacy of exercise to modulate emotional context or sad emotion regulatory capabilities in women with PMS.
\end{abstract}

Keywords: Exercise, Premenstrual Syndrome, Brain, Sad Emotion, Electroencephalography

\section{Introduction}

Premenstrual syndrome (PMS) has a high morbidity level, reaching 30-80\% among women at reproductive age $[1,2]$. PMS typically refers to the physical, psychological, and behavioral symptoms that occur in the days preceding the menses. Indeed, women with PMS experience more emotional suffering and negative mood symptoms. Moreover, the incidence of depression among women with PMS is greater than that of the general population $[3,4]$. Premenstrual dysphoric disorder (PMDD) is a particularly severe and disabling form of PMS identified in previous clinical studies, which affects $3-8 \%$ of women $[5,6]$.

Recently, neuroimaging studies have focused attention on the high prevalence of PMS. These studies have indicated abnormal spontaneous brain activity in women with PMS, 
with the severity of symptoms specifically related to left medial frontal cortex (MFC) and right anterior cingulated cortex (ACC) activity [7]. Furthermore, women with PMS displayed abnormal resting-state connectivity, with clearly decreased connectivity in the middle frontal gyrus (MFG) and para-hippocampal gyrus (PHG) [8], regions known to play a role in emotional executive functions. Historically, it has been assumed that emotional cognitive control is primarily regulated by prefrontal regions [9]. These regions serve the highest cognitive functions, optimizing and scheduling lower-order functions via links with broadly dispersed regions of the limbic cortices [10]. In this study, we hope to gain insight into emotional executive functions in women with PMS.

Physical activity is associated with improved affective experience and enhanced cognitive processing. Both clinical and non-clinical participants have been shown to benefit acutely from even a single bout of exercise [11-13]. There are multiple mechanisms that may account for brain plasticity following exercise, including mobilization of neurochemical expression, increased production of BDNF, increased cerebral blood flow, and increased oxygen delivery [17, 18], which can act to protect against neurological disorders [11, 16].

Converging studies have revealed that frontal neural circuits play a crucial role in mediating exercise-related changes to cognitive function and emotion processing [11, 14, 15]. Exercise may serve as a protective factor in individuals with negative affect who have recovered from depression, as shown within a repeated sad mood induction paradigm [16]. Additionally, acute exercise is one of the most effective behavioral techniques for self-regulation of mood in healthy populations [17]. Observational studies have reported the effects of exercise on relieving symptoms of PMS [18]. Nevertheless, the effects of exercise on neural processing of negative emotions, such as sadness (i.e., sad emotion processing), specifically in women with PMS remain poorly understood.

The ability to effectively regulate emotions, such as sadness, plays an important role in facilitating social interactions and mental well-being [19]. Event-related potentials (ERPs) provide a non-invasive and convenient technique to measure brain activity in response to various stimuli, and have been extensively used to explore cognitive and emotion processing for research and clinical purposes [20].

Emotions can be elicited by external stimuli and previous research has identified several ERP components that are reliably elicited by facial stimuli during experimental manipulations [21-24]. One particular ERP component, $\mathrm{N} 250$, is a negative deflection that peaks approximately 250 msec after stimulus presentation and reflects complex, detailed processing of facial stimuli, including that of facial emotions $[25,26]$. The fronto-centrally distributed N250 has been proposed to underlie decoding of emotional cues, such as the extraction of emotional cues from faces [27-30]. Furthermore, N250 is considered to be a marker of higher- order face processing, such as affect decoding, and is presumed to reflect modulatory influences of subcortical structures, including the amygdala $[31,32]$. ERPs provide evidence for a sequential pattern of brain activation underlying task-related information processing and can provide a sensitive indicator of the effects of exercise on PMS.

The effects of exercise on ERPs produced in response to higher-order face processing of sad stimuli in fronto-central brain regions of women with PMS remain unclear. As mentioned above, brain activity is modulated by PMS severity [8]. Meanwhile, exercise can mediate cognitive abilities and promote women's health [18] by fine-tuning neuroplasticity $[33,34]$. In this study, we conducted an eventrelated analysis of target stimuli demonstrating time-locked activation associated with correct identification of sad faces. By using ERPs, we evaluated potentially different effects of exercise on neural activity correlated with sad emotion processing in women with PMS of varying severity (i.e., high-PMS, HMPS, and low-PMS, LPMS groups).

The aim of the study was to investigate the hypothesis that the modulatory effects of exercise on sad emotion processing reflected by fronto-central neural activation differ between HPMS and LPMS.

\section{Methods}

\subsection{Participants}

We recruited paid female volunteers $(n=31$, all righthanded), aged 18-22 years old (mean age: 24.4 years). Participants were excluded if they: (1) had a history of neurological or psychiatric disorders; (2) had chronic cardiorespiratory illnesses; (3) had limb movement disorder; (4) used cortisol medications; (5) were diagnosed with a personality disorder; (6) were athletes; (7) had regularly engaged in intensive exercise during the past three months; or (8) used oral contraceptive pills (OCPs) in the past year. Participants were also screened to exclude women with PMDD (evaluated with the DSM-IV). Participants were asked to refrain from alcohol for 48 hours and caffeine for 12 hours before the day of the experiment

All experimental procedures were performed in accordance with appropriate guidelines and were approved by the institutional review board of Chang Gung Memorial Hospital, Taiwan. All participants provided written informed consent prior to the experiment.

\subsection{Physical Activity Assessment}

Each volunteer was asked to ride an exercise bike for 20 minutes after finishing the first ERP recording. Participants were then instructed to perform aerobic fitness on a treadmill for $20 \mathrm{~min}$ with a consistent speed of around 25.6-28.8 mph (16-18 km/hr). Graded practice was preceded by a warm-up ( $5 \mathrm{~min}, 1 \%$ grade), followed by $5 \mathrm{~min}$ each at grades $3 \%$ and $4 \%$, with a final $5 \mathrm{~min}$ cool-down at grade $1-2 \%$. Trained nurses ensured that experimental procedures were safely 
conducted throughout.

Each participant underwent ERP recordings on two occasions. The first test was conducted before physical activity. The second test was conducted during rest, $90 \mathrm{~min}$ after the end of physical activity.

\subsection{Facial Expression Recognition Task}

We conducted a facial expression recognition task to examine variations in frontal-central brain activation during cognitive responses of our participants. Facial expression stimuli were black and white pictures in which the emotion indicated by the facial expression could be clearly distinguished. The pictures were taken from a standard set of pictures of facial affect (Ekman emotion photos) and were presented in the middle of a computer screen on a black background. Participants were shown a 500-ms fix-cross picture as a warning signal, followed $200 \mathrm{~ms}$ later by a 900 ms facial expression picture.

After the instructor introduced the experiment, the 96 monochrome Ekman human facial emotion photos, including 24 for each emotion (happy, neutral, sad, and fear), were presented in a random order on the screen. Each volunteer was asked to silently count the number of sad emotion pictures presented and to report the number after the task in each ERP recording. This ensured that participants paid attention and concentrated during the task.

Importantly, our study design considered the rapid habituation of amygdala responses to facial expressions of emotion apparent at high stimulation frequencies [35]. Furthermore, all protocols were piloted and established prior to the official experiment, which revealed that participants sometimes feel sleepy during a long experimental process in a dark room after exercise. Therefore, we limited each ERP recording session to 5-6 $\mathrm{min}$.

\subsection{ERP Recordings}

For ERP recordings, participants were asked to rest for 90 min. During the recording process, each volunteer sat in front of a screen in a dark room. Participants were asked to sit comfortably on a chair and ERP electrodes were fixed to the scalp as per the International 10-20 electrode placement system.

Electroencephalography (EEG) signals were collected with Encephalan-EEG software (Medicom MTD, Taganrog, Russia). Data were recorded from 19 sites: Fp1, Fp2, F3, F4, F7, F8, Fz, C3, C4, Cz, T3, T4, T5, T6, P3, P4, Pz, O1, and $\mathrm{O} 2$ by using the standard 10-20 system, with a sampling rate of 250 per sec. We used high and low filters of 70 and $0.1 \mathrm{~Hz}$ and a rejecter of $60 \mathrm{~Hz}$, with impedances below $10 \mathrm{k} \Omega$.

\subsection{PMS Evaluation}

We administered a PMS self-report questionnaire, the PMS Inventory, developed by Steiner et al [36]. The questionnaire has been modified and used to screen for PMS severity in both clinical and nonclinical settings with adolescents in sequential studies. The inventory has 14 descriptions related to physical, psychological, and behavioral symptoms. Participants read the descriptions and score each item from 1 (low) to 5 (high), according to their perceived condition. Therefore, higher scores indicate more severe PMS, with total scores ranging from 14 to 70. According to scores on the PMS Inventory, participants were divided into two groups: HPMS and LPMS. We then analyzed cortical activity in response to sad cues, and compared activity between the two groups.

\subsection{Statistical Analysis}

\subsubsection{Behavioral Data}

We evaluated descriptive statistics, including means and standard deviations (SDs; mean \pm SD) of the PMS inventory scores. Participants reported the number of sad pictures observed during each period (pre- and post-exercise), and data were compared with two-tailed paired $t$-tests.

\subsubsection{ERP Component}

We recorded and analyzed ERP components evoked by sad faces at 19 channels during pre- and post-exercise sessions. Based on the aforementioned time windows of evoked responses (see Introduction), the component peak amplitude inspection was extracted for each participant in each condition, and the average of all channels was calculated. Once selected, latency windows were the same for all participants and conditions. As expected, a prominent peak was identified in the $230-290 \mathrm{msec}$ time window (N250) at the fronto-central channel during sad stimuli.

To calculate a grand mean value of N250, a two-way, repeated-measures ANOVA was conducted for the main effects of exercise (pre- and post-exercise) and group (LPMS and HPMS) on the N250 component at each electrode location. Where the ANOVA yielded a significant main effect at the $P<$ 0.05 level, successive single comparisons using $t$-tests were conducted. We selected 10 fronto-central channels including the frontal pole (Fp1, Fp2), medial frontal $(\mathrm{F} 3, \mathrm{~F} 4)$, lateral frontal $(\mathrm{F} 7, \mathrm{~F} 8)$, central $(\mathrm{C} 3, \mathrm{C} 4)$, and fronto-central midline $(\mathrm{Fz}, \mathrm{Cz}$ ) electrodes because activity in these regions is specifically related to cognitive processing of sad emotions.

The independent variables included group (HPMS or LPMS) and period (pre- and post-exercise). The dependent variables were the amplitudes of $\mathrm{N} 250$ at each channel (Fp1, Fp2, F3, F4, F7, F8, Fz, C3, C4, and Cz). We performed independent pooled $t$-tests to compare differences in N250 between HPMS and LPMS during the two periods. A paired $t$-test was conducted to evaluate differences in the $\mathrm{N} 250$ component (amplitude and latency) within groups between the two periods. For all analyses, the significance level was set at $P<0.05$, two-tailed. All statistical analyses were conducted with SPSS 18 (IBM Corp., Armonk, NY).

\subsubsection{Correlation of ERP Component and PMS Score}

The correlation between ERP activation (amplitude, latency) and PMS assessment was examined. The relationships between mean amplitude or latency and PMS scores were analyzed for all participants $(n=31)$ in each session (pre- and post-exercise) with Pearson correlation. 


\section{Results}

\subsection{PMS Assessment}

The mean total PMS score in all 31 subjects was $32.61 \pm$ 8.63. Based on the distribution of PMS scores, participants were assigned to the LPMS (score $\leq 30$ ) or HPMS (score $>$ 30) group for subsequent comparisons. The mean total PMS score in the HPMS group was $39.06 \pm 6.56(\mathrm{~N}=16)$, and in the LPMS group was $25.73 \pm 3.95(\mathrm{~N}=15)$. The mean number of sad pictures reported after EEG recording did not differ between the two groups for each period $(P>0.05)$ (pre- and post-exercise).

\subsection{ERP: Sad Facial-Evoked N250}

We observed a significant effect of group on N250 amplitude. As such, the mean value of the N250 amplitude was significantly different at Fp1, Fp2, F3, Fz, F4, F8, C3, $\mathrm{Cz}$, and $\mathrm{C} 4(F[1,31]=25.24,11.09,15.07,37.52,11.98$, $8.80,23.03,11.53$, and 15.18, respectively; LPMS vs. HPMS, $P<0.05$; Greenhouse-Geiser corrected; Table 1). We also observed a significant difference in the mean value of the $\mathrm{N} 250$ latency at $\mathrm{F} 7, \mathrm{~F} 3$, and $\mathrm{C} 4(F[1,31]=7.22,4.86$, and 6.65, respectively; LPMS vs. HPMS, $P<0.05$; Table 1$)$.

Table 2 (left and middle column) presents successive pairwise comparisons of N250 activation in LPMS and
HPMS groups in pre- and post-exercise sessions. This analysis suggested no differences in activation between preand post-exercise sessions in the LPMS group. Conversely, there was a significant attenuation of N250 amplitude postexercise compared with pre-exercise at almost every channel in the HPMS group $(P<0.05$; Table 2 ; Figure 1,2$)$. The N250 latency for processing of sad stimuli in the HPMS group was significantly prolonged in the post-exercise condition compared with pre-exercise at F3 and $\mathrm{C} 3(P<$ $0.05)$. However, there were no significant differences in N250 latency between the two conditions at any channel in the LPMS group.

Table 2 also presents successive pooled $t$-tests comparing N250 activation to sad stimuli, which shows that there was no significant difference between the two groups at baseline. Nevertheless, we controlled for baseline N250 activation using the mixed models procedure for between group differences. Subsequently, we observed a significant difference in N250 activation between the groups after exercise. After exercising, there was a significant reduction in the N250 brain response in the HPMS group at F7 and F8 ( $t$ $[29]=-2.78$ and -2.16 , respectively; LPMS vs. HPMS, $P<$ $0.05)$. Our data suggest that the observation of differences in neural plasticity elicited by exercise during sad emotion recognition depends on severity of PMS.

Table 1. Repeated ANOVA for the effects of aerobic exercise on the N250 component in 31 healthy women with low or high premenstrual syndrome (PMS) scores during sad facial recognition.

\begin{tabular}{|c|c|c|c|c|c|c|c|}
\hline \multicolumn{2}{|c|}{ Amplitude } & \multirow{2}{*}{$\frac{\mathbf{F}}{.273}$} & \multirow{2}{*}{$\frac{P \text { value }}{.61}$} & \multicolumn{2}{|c|}{ Latency } & \multirow{2}{*}{$\frac{\mathbf{F}}{.682}$} & \multirow{2}{*}{$\frac{P \text { value }}{.424}$} \\
\hline EP1 & LPMS & & & & LPMS & & \\
\hline ГT1 & HPMS & 25.235 & $.000 * * *$ & 111 & HPMS & 1.818 & .201 \\
\hline \multirow{2}{*}{ FP2 } & LPMS & .454 & .512 & \multirow{2}{*}{ FP2 } & LPMS & .005 & .944 \\
\hline & HPMS & 11.086 & $.005^{* *}$ & & HPMS & .124 & .73 \\
\hline \multirow{2}{*}{ F7 } & LPMS & 1.368 & .263 & \multirow{2}{*}{ F7 } & LPMS & 7.216 & $.019 *$ \\
\hline & HPMS & 2.589 & .132 & & HPMS & 4.419 & .056 \\
\hline \multirow{2}{*}{ F3 } & LPMS & .876 & .366 & \multirow{2}{*}{$\mathrm{F} 3$} & LPMS & .804 & .386 \\
\hline & HPMS & 15.074 & $.002 * *$ & & HPMS & 4.864 & $.046^{*}$ \\
\hline \multirow{2}{*}{$\mathrm{Fz}$} & LPMS & 1.074 & .319 & \multirow{2}{*}{$\mathrm{Fz}$} & LPMS & .069 & .797 \\
\hline & HPMS & 37.515 & $.000 * * *$ & & HPMS & 3.1 & .102 \\
\hline \multirow{2}{*}{ F4 } & LPMS & .645 & .436 & \multirow{2}{*}{$\mathrm{F} 4$} & LPMS & .067 & .8 \\
\hline & HPMS & 11.976 & $.004 * *$ & & HPMS & 4.263 & .059 \\
\hline \multirow{2}{*}{ F8 } & LPMS & 1.144 & .304 & \multirow{2}{*}{ F8 } & LPMS & .053 & .822 \\
\hline & HPMS & 8.795 & $.011 *$ & & HPMS & .887 & .363 \\
\hline \multirow{2}{*}{$\mathrm{C} 3$} & LPMS & .896 & .361 & \multirow{2}{*}{$\mathrm{C} 3$} & LPMS & .944 & .349 \\
\hline & HPMS & 12.06 & $.004 * * *$ & & HPMS & 2.105 & .171 \\
\hline \multirow{2}{*}{$\mathrm{Cz}$} & LPMS & .314 & .585 & \multirow{2}{*}{$\mathrm{Cz}$} & LPMS & .004 & .951 \\
\hline & H PMS & 23.032 & $.000 * * *$ & & HPMS & 4.12 & .063 \\
\hline \multirow{2}{*}{$\mathrm{C} 4$} & LPMS & 1.602 & .2 & \multirow{2}{*}{$\mathrm{C} 4$} & LPMS & .003 & .957 \\
\hline & HPMS & 11.525 & $.005^{* *}$ & & HPMS & 6.648 & $.023^{*}$ \\
\hline
\end{tabular}

$* \mathrm{P}<.05 ; * * \mathrm{P}<.01 ; * * * \mathrm{P}<.001$ 
Table 2. The N250 amplitudes between low premenstrual syndrome (LPMS) and high premenstrual syndrome (HPMS) groups.

\begin{tabular}{|c|c|c|c|c|c|c|c|c|c|}
\hline & & \multicolumn{6}{|c|}{ Low PMS Group Mean Differences } & \multicolumn{2}{|c|}{ High PMS Group Mean Differences } \\
\hline & & $\begin{array}{l}\text { Baseline } \\
\text { mean }\end{array}$ & $\pm \mathbf{S E}$ & $\begin{array}{l}\text { Post-Exercise } \\
\text { mean }\end{array}$ & $\pm \mathbf{S E}$ & $\begin{array}{l}\text { Mean } \\
\text { Difference }\end{array}$ & $t(d f=14)$ & Baseline mean & $\pm \mathbf{S E}$ \\
\hline \multirow{2}{*}{ Fp1 } & Amp & -4.63 & 0.75 & -3.73 & 0.83 & -0.9 & -1.23 & -5.73 & 0.77 \\
\hline & Latency & 238.67 & 7.65 & 240.8 & 8.04 & -2.13 & -0.35 & 230 & 7.81 \\
\hline \multirow{2}{*}{ Fp2 } & Amp & -4.53 & 0.77 & -3.52 & 0.91 & -1.01 & -1.03 & -5.55 & 0.85 \\
\hline & Latency & 231.47 & 7.6 & 241.33 & 8.48 & -9.87 & -1.08 & 248.5 & 9.59 \\
\hline \multirow[b]{2}{*}{ F7 } & Amp & -3.71 & 0.76 & -4.2 & 0.66 & 0.49 & 0.61 & -3.97 & 0.52 \\
\hline & Latency & 241.33 & 8.36 & 250.4 & 7.54 & -9.07 & -1.16 & 221.5 & 6.25 \\
\hline \multirow{2}{*}{$\mathrm{F} 3$} & Amp & -4.53 & 0.68 & -4.49 & 0.92 & -0.03 & -0.06 & -4.84 & 0.49 \\
\hline & Latency & 246.67 & 7.85 & 251.73 & 6.86 & -5.07 & -0.65 & 230.25 & 8.05 \\
\hline \multirow{2}{*}{$\mathrm{Fz}$} & Amp & -5.31 & 0.65 & -4.18 & 0.99 & -1.13 & -1.9 & -5.06 & 0.64 \\
\hline & Latency & 236 & 7.54 & 247.73 & 7.56 & -11.73 & -1.81 & 238.25 & 9.03 \\
\hline \multirow{2}{*}{ F4 } & Amp & -4.53 & 0.63 & -3.71 & 0.93 & -0.83 & -1.09 & -4.61 & 0.54 \\
\hline & Latency & 237.07 & 7.43 & 246.13 & 7.74 & -9.07 & -1.31 & 232.25 & 8.33 \\
\hline \multirow{2}{*}{ F8 } & Amp & -4.6 & 0.67 & -3.69 & 0.74 & -0.91 & -1.15 & -4.33 & 0.51 \\
\hline & Latency & 235.73 & 7.73 & 242.4 & 7.48 & -6.67 & -1.1 & 242 & 8.93 \\
\hline \multirow[b]{2}{*}{$\mathrm{C} 3$} & Amp & -2.98 & 0.65 & -3.31 & 0.98 & 0.33 & 0.59 & -3.59 & 0.43 \\
\hline & Latency & 245.6 & 6.54 & 244.8 & 6.25 & 0.8 & 0.1 & 226.75 & 7.03 \\
\hline \multirow{2}{*}{$\mathrm{Cz}$} & Amp & -3.63 & 0.74 & -3.06 & 1.06 & -0.57 & -0.87 & -4.32 & 0.54 \\
\hline & Latency & 240.8 & 6.76 & 248.53 & 6.99 & -7.73 & -1.02 & 240.25 & 9.17 \\
\hline \multirow{2}{*}{$\mathrm{C} 4$} & Amp & -3.63 & 0.84 & -3.07 & 1.01 & -0.57 & -0.94 & -3.39 & 0.43 \\
\hline & Latency & 234.93 & 6.92 & 247.2 & 7.7 & -12.27 & -1.62 & 236 & 8.09 \\
\hline
\end{tabular}

Table 2. Continued.

\begin{tabular}{|c|c|c|c|c|c|c|c|c|c|}
\hline & & \multirow{2}{*}{\multicolumn{4}{|c|}{ High PMS Group Mean Differences }} & \multirow{2}{*}{\multicolumn{4}{|c|}{$\begin{array}{l}\text { Between Group } \\
\text { Mean Differences (MD) } \\
\end{array}$}} \\
\hline & & & & & & & & & \\
\hline & & \multirow{2}{*}{$\begin{array}{l}\text { Post-Exercise } \\
\text { mean }\end{array}$} & \multirow{2}{*}{ \pm SE } & \multirow{2}{*}{$\begin{array}{l}\text { Mean } \\
\text { Difference }\end{array}$} & \multirow{2}{*}{$t(d f=15)$} & \multicolumn{2}{|l|}{ Pre } & \multicolumn{2}{|l|}{ Post } \\
\hline & & & & & & MD & $t(d f=29)$ & MD & $t(d f=29)$ \\
\hline \multirow{2}{*}{ Fp1 } & Amp & -2.05 & 0.85 & -3.68 & $-3.98 * *$ & 1.1 & 1.018 & -1.68 & -1.405 \\
\hline & Latency & 240.25 & 9.98 & -10.25 & -1.41 & 8.67 & 0.792 & 0.55 & 0.043 \\
\hline \multirow{2}{*}{ Fp2 } & Amp & -1.56 & 0.8 & -3.98 & $-3.78 * *$ & 1.02 & 0.888 & -1.95 & -1.62 \\
\hline & Latency & 238 & 8.88 & 10.5 & 0.89 & -17.03 & -1.38 & 3.33 & 0.271 \\
\hline \multirow{2}{*}{ F7 } & Amp & -1.85 & 0.53 & -2.12 & $-4.54 * * *$ & 0.26 & 0.286 & -2.35 & $-2.782 *$ \\
\hline & Latency & 234.75 & 9.34 & -13.25 & -1.47 & 19.83 & 1.916 & 15.65 & 1.293 \\
\hline \multirow{2}{*}{ F3 } & Amp & -2.13 & 0.6 & -2.7 & $-4.24 * * *$ & 0.31 & 0.375 & -2.36 & -2.174 \\
\hline & Latency & 257.25 & 9.91 & -27 & $-2.51 *$ & 16.42 & 1.457 & -5.52 & -0.458 \\
\hline \multirow{2}{*}{$\mathrm{Fz}$} & Amp & -2.09 & 0.71 & -2.97 & $-3.44 * *$ & -0.25 & -0.269 & -2.09 & -1.734 \\
\hline & Latency & 246.75 & 9.76 & -8.5 & -0.89 & -2.25 & -0.19 & 0.98 & 0.079 \\
\hline \multirow{2}{*}{ F4 } & Amp & -2.13 & 0.67 & -2.48 & $-3.02 * *$ & 0.08 & 0.092 & -1.58 & -1.393 \\
\hline & Latency & 252.5 & 8.95 & -20.25 & -1.97 & 4.82 & 0.429 & -6.37 & -0.535 \\
\hline \multirow{2}{*}{ F8 } & Amp & -1.59 & 0.63 & -2.73 & $-3.75 * * *$ & -0.27 & -0.326 & -2.1 & $-2.158^{*}$ \\
\hline & Latency & 247 & 7.9 & -5 & -0.48 & -6.27 & -0.527 & -4.6 & -0.422 \\
\hline \multirow{2}{*}{$\mathrm{C} 3$} & Amp & -1.24 & 0.48 & -2.34 & $-4.69 * * *$ & 0.6 & 0.77 & -2.07 & -1.898 \\
\hline & Latency & 249.5 & 9.58 & -22.75 & $-2.35^{*}$ & 18.85 & 1.956 & -4.7 & -0.411 \\
\hline \multirow{2}{*}{$\mathrm{Cz}$} & Amp & -1.38 & 0.6 & -2.94 & $-4.96 * * *$ & 0.7 & 0.765 & -1.68 & -1.401 \\
\hline & Latency & 255.25 & 9.62 & -15 & -1.92 & 0.55 & 0.048 & -6.72 & -0.565 \\
\hline \multirow{2}{*}{$\mathrm{C} 4$} & Amp & -1.38 & 0.6 & -2.01 & $-3.08 * *$ & -0.24 & -0.263 & -1.68 & -1.454 \\
\hline & Latency & 252.25 & 8.36 & -16.25 & -1.9 & -1.07 & -0.1 & -5.05 & -0.443 \\
\hline
\end{tabular}

LPMS = low premenstrual syndrome; HPMS = high premenstrual syndrome; $\mathrm{MD}=$ mean difference; $\mathrm{SE}=\mathrm{standard}$ error. $* \mathrm{P}<.05 ; * * \mathrm{P}<.01 ; * * * \mathrm{P}<.001$ 


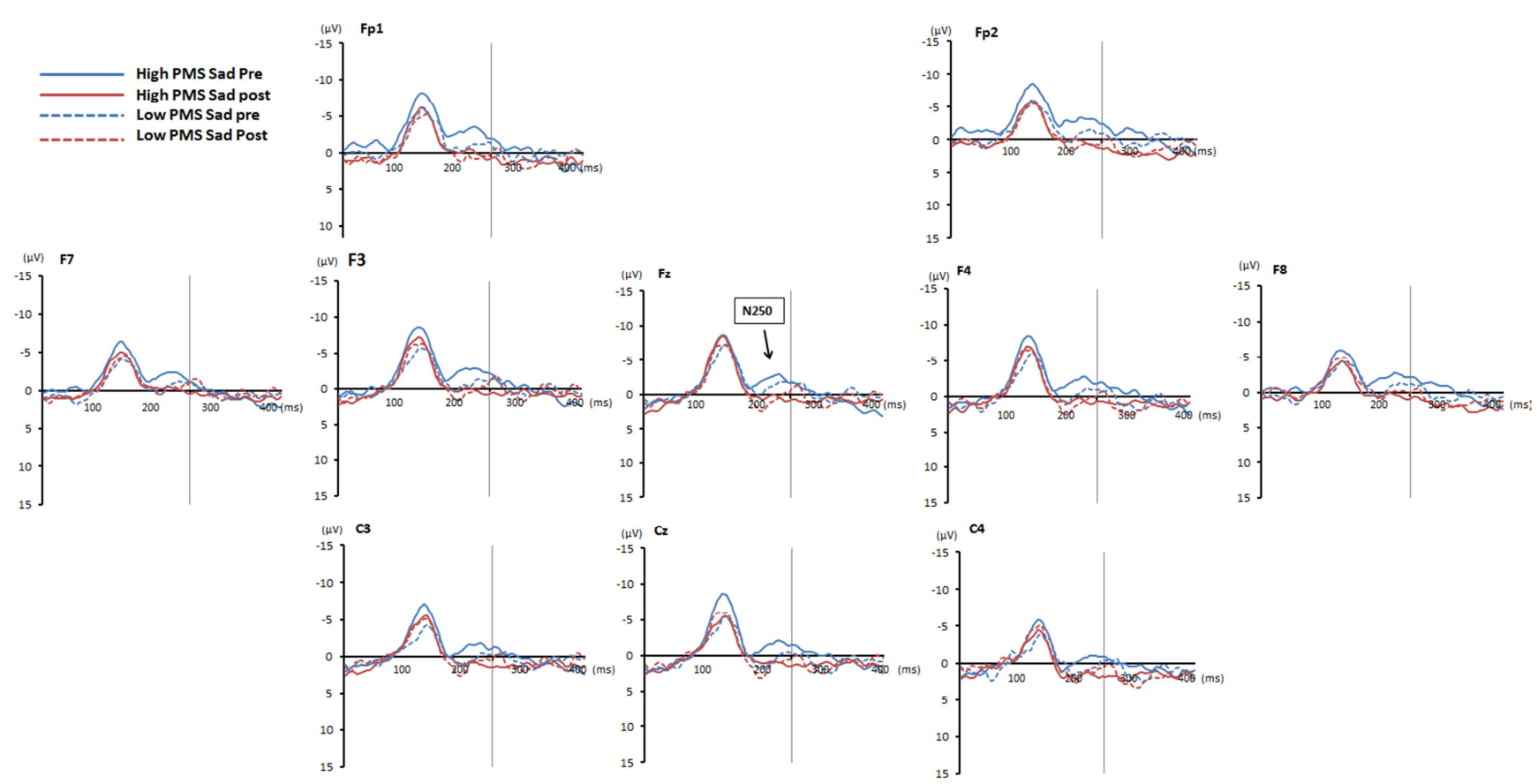

Figure 1. N250 event-related potential component, a negative peak at 200-300ms after stimuli presentation, was evaluated over the anterior frontal region. $P M S=$ premenstrual syndrome; Pre $=$ pre-exercise condition; Post $=$ post-exercise condition.
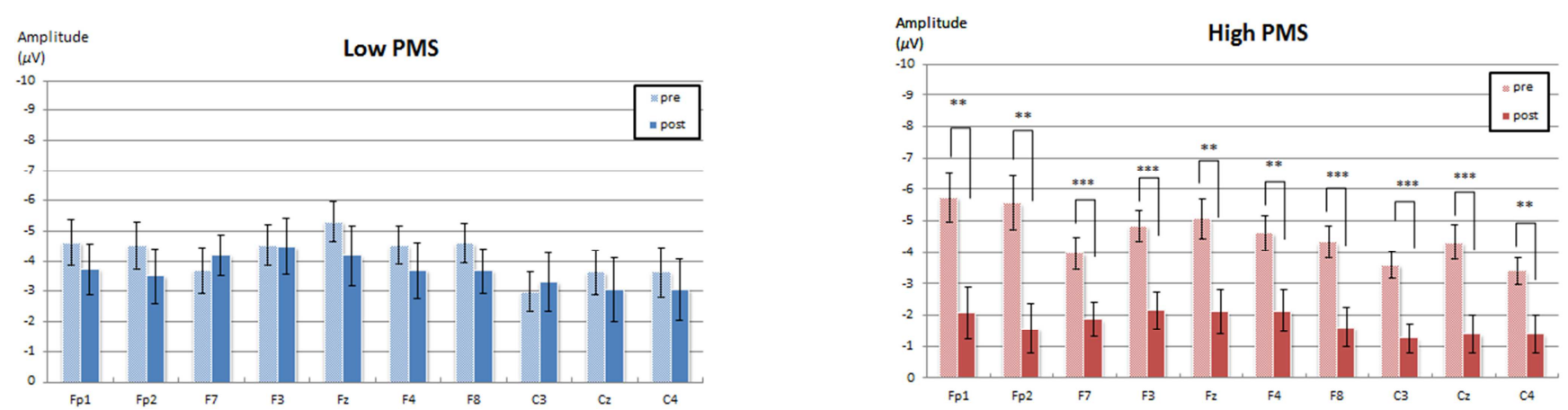

Figure 2. The amplitude of $N 250$ in response to sad emotional stimuli in high and low premenstrual syndrome (PMS) groups modulated by exercise

Exercise was associated with significantly lower N250 amplitudes at Fp1, Fp2, Fz, F4, and C4 $(P<0.01)$ and at $F 7, F 3, F 8, C 3$, and $C z(P<0.001)$ compared with preexercise in the high PMS group. However, this effect was not seen in the low PMS group. PMS = premenstrual syndrome; pre $=$ pre-exercise condition; post $=$ post-exercise condition .

\subsection{Correlation Between the PMS Score and N250 Component}

There were significant negative correlations between PMS scores and N250 amplitude at Fp1, Fp2, and Fp3 channels during the pre-exercise session $(r=-.445,-.377,-.355$, respectively; all $P<0.05, \mathrm{n}=31$ ). These results suggest that women with higher PMS scores had greater N250 amplitudes at these channels. However, this association was not observed for post-exercise measurements (Table 3). There were no significant relationships between PMS scores and N250 latency at any other channel in either condition (all $P>$ $0.05)$.

Table 3. Correlations between PMS score and the N250 component modulated by exercise $(n=31)$.

\begin{tabular}{|c|c|c|c|c|c|c|c|c|c|c|c|}
\hline & & Fp1 & Fp2 & F7 & F3 & $\mathbf{F z}$ & F4 & F8 & $\mathrm{C3}$ & $\mathrm{CZ}$ & $\mathrm{C} 4$ \\
\hline \multirow{2}{*}{ Pre-exercise } & $r$ & $-.445^{*}$ & $-.377 *$ & -0.252 & $-.355^{*}$ & -0.278 & -0.292 & -0.176 & -0.268 & -0.273 & -0.11 \\
\hline & $p$ & 0.012 & 0.037 & 0.171 & 0.05 & 0.13 & 0.111 & 0.342 & 0.145 & 0.137 & 0.555 \\
\hline \multirow{2}{*}{ Post-exercise } & $r$ & 0.1 & 0.106 & 0.262 & 0.143 & 0.121 & 0.14 & 0.238 & 0.198 & 0.133 & 0.24 \\
\hline & $p$ & 0.591 & 0.571 & 0.155 & 0.444 & 0.516 & 0.453 & 0.198 & 0.285 & 0.476 & 0.193 \\
\hline
\end{tabular}




\section{Discussion}

No previous studies have explored the neurobiology underlying how exercise interacts with processing of sad emotional stimuli in women with PMS of varying severity. Therefore, the objective of the current study was to explore relationships between exercise and higher order cognitive processing of sad emotions in women with PMS.

Our findings suggest that the impact of aerobic exercise on fronto-central responses to sad facial expressions depends on PMS severity. We observed that the fronto-central cortex of HPMS participants exhibited more changes in exerciserelated cognitive resource allocation to task-relevant stimuli than this that of LPMS participants (Table 1). N250 activation evoked by sad emotions did not differ between the groups at baseline. However, there was a significant reduction in N250 activation in the lateral prefrontal cortex (PFC) of the HPMS group compared with the LPMS group after exercise (channels F7 and F8; $P<.05$ ). Furthermore, an exercise-mediated association between PFC-N250 power and the PMS score $(P<0.01)$ was observed (Table 3). Our results support the suggestion that affective neural circuit alterations are dependent on PMS severity and susceptible to exercise modulation for HPMS. Neural flexibility observed following exercise supports a potentially effective intervention to modulate the neural substrate of sad emotional regulatory capabilities in women with PMS.

Exercise-related brain activity in response to sad stimuli in PMS

Previous ERP studies of the time course of emotional face processing have reported that $\mathrm{N} 250$ is elicited by faces in emotion recognition tasks [25-30], playing a role in decoding of emotional facial expressions [37]. It appears that N250 is particularly related to negative emotion processing at electrodes distributed across fronto-central regions [38].

Here, we observed N250 activation evoked by sad emotions that peak within 200-300 msec following stimulus onset. However, exercise modified neurocognitive processing of sad stimuli, with differences observed between HPMS and LPMS groups. In the HPMS group, N250 amplitude was significantly attenuated post-exercise compared with preexercise at most fronto-central regions. However, this pattern was not observed in the LPMS group (Table 2; Figures 1, 2). We also observed variations in the latency of N250 to sad stimuli, with a significantly longer latency post-exercise compared with pre-exercise at $\mathrm{C} 3$ and $\mathrm{F} 3$ in HPMS $(P<.05$; Table 2).

EEG has the advantage of providing coherent information about neural activity in the form of latency and amplitude. It appears that exercise-mediated effects on these neural indices of responses to sad stimuli (reduced amplitude and increased latency) may occur in HPMS but not in LPMS. The frontocentral cortex is involved in both the generation and regulation of emotion via various subcortical pathways [9]. Exercise-induced neuroplastic changes to modulate affective processing has previously been observed with both long-term and acute exercise $[39,40]$. The effects of acute bouts of exercise on emotional cognitive function have previously been documented [16, 41, 42]. Our findings provide further support, but suggest that the underlying mechanism may be more complex, such that the severity of PMS determines the effectiveness of exercise to modulate emotion processing. This suggests that exercise can potentially affect the emotional context or sad emotion regulatory capabilities of women with HPMS.

We observed that acute exercise visibly enhanced brain plasticity in response to sad emotions in HPMS compared with LPMS (Table 1). This finding can be explained in several ways. PMS is thought to be caused by an underlying neurobiological vulnerability to normal fluctuations in circulating sex hormones, with estrogen and progesterone receptors expressed in the nucleus accumbens, a terminal region of the mesolimbic dopaminergic system [43-45]. Exercise facilitates the brain's functional capabilities with the release of BDNF via the serotonin-BDNF loop [11], together with involvement of mesolimbic dopaminergic system modulation [46, 47]. Furthermore, neuroimaging studies have indicated abnormal resting-state connectivity of frontallimbic regions in women with PMS who displayed abnormal stress sensitivity [8]. Physical activity is particularly effective at mediating these affective neural circuits and related moderation of psychological symptoms [48-50]. The circumstances under which neural networks are complexly and dynamically modulated by exercise depend on PMS severity. It is possible that HPMS participants are more susceptible to neurogenesis and neurotransmitter regulation caused by exercise than are women with less severe PMS.

Our results suggest that, based on PMS severity, acute exercise enhances neural plasticity, thus enhancing cognitive processing of sad emotion stimuli. This may reflect differences in emotional self-efficacy. Humans can successfully cope with mild to moderate stressors via neurochemical factors that are considered to be essential contributors to brain resilience in challenging circumstances $[51,52]$. Our participants were healthy women, screened to exclude a history of neurological and psychiatric disorders, and PMDD (see Methods), with a mean PMS score of 34 (range: 14-70). Behavioral studies have shown that the ability of moderate aerobic exercise to attenuate negative emotions varies depending on human behavioral responses [41]. Emotion regulation is an automatic or deliberate use of strategies to initiate, maintain, modify, or display emotions $[53,54]$. An exercise-induced transient state of frontal hypofunction coordinates autonomic regulation of psychological states [55]. In our study, sad stimuli induced attenuation of evoked N250 after exercise compared with baseline. Thus, this may conceivably reflect brain resilience and an adaptation to intrinsic demands for a purposeful, finetuned, and flexible approach in the women with HPMS

Exercise mediates lateral PFC activity depending on PMS severity

The within group comparisons outlined above suggest that only HPMS participants typically showed more flexible and resilient neural function in the fronto-central cortex following 
exercise. In contrast, between groups analysis suggested that there were no differences in N250 activation to sad stimuli between the two groups at baseline. However, significantly lower N250 activation was observed at F7 and F8 in individuals with HPMS compared with LPMS after exercise.

It is possible to map the topography of the brain to different functions. Indeed, EEG-based functional connectivity is taken to reflect the relationship between brain activity and emotional states [56]. Electrodes at F7 and F8 are located near the lateral PFC, and the process of cognitive emotion regulation is functionally organized within the lateral PFC [57]. For example, F7 is related to avoidance behavior and impulse control (with important links to the amygdala) [58]. Meanwhile, F8 activity enables emotional regulation and induces approach behavior. Therefore, the lateral PFC contributes to emotion regulation and is a critical neural substrate involved in representing the emotional value of engaging in cognitive control [59-62].

Our results suggest a significant difference in N250 activation at the lateral PFC between the groups after exercise, while no significant difference was observed at baseline. Studies documenting exercise-mediated changes in the emotional regulation system have suggested there is intrinsic tuning of one's emotional state. The requirement for modification of the resources ascribed to cognitive processing may reflect an innate physiological response accompanying different levels of autonomic arousal between the groups after exercise. The lateral PFC is involved in emotion regulation and modulates activity in subcortical emotion-generating systems that require effortful processes to alter individual emotional states [9, 63]. Moreover, the impact of physical exercise-induced arousal or attention varies depending on the underlying emotion [59]. We observed that the process of effortful emotional regulation is mediated differently by exercise between the groups. Furthermore, the brain resources that are made available after exercise also appear to differ between the groups. This may translate into better emotional neurocognitive efficiency or function for HPMS participants. The observation that exercise mediates the neural basis of sadness intensity or valence in PMS should be further explored in future research.

Correlations between N250 and PMS scores

Our correlation analysis revealed that, before exercise, women with higher PMS scores displayed greater N250 amplitude at the PFC in response to sad emotion stimuli (Fp1, Fp2 and Fp3 electrode sites; all $P<0.05$ ) (Table 3). This correlation was not observed after exercise (all $P>0.05$; $\mathrm{n}=31$ ). Previous studies suggest that emotional cognitive control can be defined as a set of higher-order functions that optimize and schedule lower-order functions.

Evidence regarding the emotional and attentional capacities of the prefrontal cortex suggests that greater activity represents higher vigilance or awareness, and is associated with anxiety or mental illness [64-66]. Furthermore, research has shown that exercise mediates several neurotransmitter processes [37], exerting anxiolytic and antidepressant effects by inhibiting excessive neural activity in PFC regions $[49,67,68]$. Our observation that there are significant negative correlations between PMS scores and sad emotion-evoked PFC responses (i.e., greater N250 amplitude) before exercise that are absent after exercise may possibly represent relatively less hypervigilance and hyperawareness to sad events in the post-exercise condition. Our results may suggest more generalized alterations to cerebral processing of negative stimuli as an inherent feature of PMS severity. Exercise mediates the relationship between PMS scores and N250 activation that provides a strong neural basis for the way in which exercise modulates PMS-related neurocognitive function in the context of sad emotional regulation, acting to prevent or reverse hypervigilance.

In summary, despite the growing number of studies examining exercise and cognition, few studies have examined these in PMS. Our study is limited by a small sample size. We also only analyzed centro-frontal N250 responses based on the specific purpose of the present study. However, future studies should be conducted to examine interactions between exercise, emotional processing, and brain activation in other regions. Furthermore, appropriate tests of laterality trends to investigate asymmetry in cerebral function should be conducted in future.

\section{Conclusions}

Women with HPMS exhibited greater susceptibility of the fronto-central cortex plasticity to exercise-related cognitive resource allocation in response to sad stimuli. Notably, exercise mediates activity of the emotional regulation system of the lateral PFC (F7 and F8), suggesting that exercise is a major component of PMS-related variations in emotional cognitive functioning. The different cortical resilience patterns to sad emotions for LPMS and HPMS women indicate the potential efficacy of exercise in modulating emotional context or sad emotion regulatory capabilities in women with PMS.

\section{Acknowledgements}

We thank the 31 participants as well as CGUST for providing administrative support. The authors would like to thank Tai-Ying Liu for statistical counseling; Yu-Ling Shih for her assistance in make aerobic exercise session is adequate and En-Zi Lin, RA for her administration support. The study was sponsored by the Ministry of Science and Technology in Taiwan (NSC 100-2410-H-255 -005 -MY2; NSC 101-2629-B-255 -001 -MY2) and Chang Gung Memorial Hospital (BMRP C52). The authors declare there are no competing interests. No potential conflict of interest relevant to this article is reported. 


\section{References}

[1] Dimmock PW, Wyatt KM, Jones PW, O'Brien PM: Efficacy of selective serotonin-reuptake inhibitors in premenstrual syndrome: a systematic review. Lancet 2000, 356 (9236): 1131-1136.

[2] A DM, K S, A D, Sattar K: Epidemiology of Premenstrual Syndrome (PMS)-A Systematic Review and Meta-Analysis Study. Journal of clinical and diagnostic research: JCDR 2014, 8 (2): 106-109.

[3] Freeman EW, Rickels K, Schweizer E, Ting T: Relationships between age and symptom severity among women seeking medical treatment for premenstrual symptoms. Psychol Med 1995, 25 (2): 309-315.

[4] Roca CA, Schmidt PJ, Altemus M, Deuster P, Danaceau MA, Putnam K, Rubinow DR: Differential menstrual cycle regulation of hypothalamic-pituitary-adrenal axis in women with premenstrual syndrome and controls. J Clin Endocrinol Metab 2003, 88 (7): 3057-3063.

[5] Duenas JL, Lete I, Bermejo R, Arbat A, Perez-Campos E, Martinez-Salmean J, Serrano I, Doval JL, Coll C: Prevalence of premenstrual syndrome and premenstrual dysphoric disorder in a representative cohort of Spanish women of fertile age. European journal of obstetrics, gynecology, and reproductive biology 2011, 156 (1): 72-77.

[6] Shah NR, Jones JB, Aperi J, Shemtov R, Karne A, Borenstein $\mathrm{J}$ : Selective serotonin reuptake inhibitors for premenstrual syndrome and premenstrual dysphoric disorder: a metaanalysis. Obstetrics and gynecology 2008, 111 (5): 1175-1182.

[7] Liao H, Pang Y, Liu P, Liu H, Duan G, Liu Y, Tang L, Tao J, Wen D, Li S et al: Abnormal Spontaneous Brain Activity in Women with Premenstrual Syndrome Revealed by Regional Homogeneity. Frontiers in human neuroscience 2017, 11: 62.

[8] Liu Q, Li R, Zhou R, Li J, Gu Q: Abnormal Resting-State Connectivity at Functional MRI in Women with Premenstrual Syndrome. PloS one 2015, 10 (9): e0136029.

[9] Ochsner KN, Gross JJ: The cognitive control of emotion. Trends in cognitive sciences 2005, 9 (5): 242-249.

[10] Miller EK, Cohen JD: An integrative theory of prefrontal cortex function. Annual review of neuroscience 2001, 24: 167202.

[11] Heijnen S, Hommel B, Kibele A, Colzato LS: Neuromodulation of Aerobic Exercise-A Review. Frontiers in psychology 2015, 6: 1890.

[12] Kelly ME, Loughrey D, Lawlor BA, Robertson IH, Walsh C, Brennan S: The impact of exercise on the cognitive functioning of healthy older adults: a systematic review and meta-analysis. Ageing Res Rev 2014, 16: 12-31.

[13] Wogensen E, Mala H, Mogensen J: The Effects of Exercise on Cognitive Recovery after Acquired Brain Injury in Animal Models: A Systematic Review. Neural plasticity 2015, 2015: 830871 .

[14] Hillman CH, Kamijo K, Scudder M: A review of chronic and acute physical activity participation on neuroelectric measures of brain health and cognition during childhood. Preventive medicine 2011, 52 Suppl 1: S21-28.
[15] Hillman CH, Snook EM, Jerome GJ: Acute cardiovascular exercise and executive control function. International journal of psychophysiology: official journal of the International Organization of Psychophysiology 2003, 48 (3): 307-314.

[16] Mata J, Hogan CL, Joormann J, Waugh CE, Gotlib IH: Acute exercise attenuates negative affect following repeated sad mood inductions in persons who have recovered from depression. J Abnorm Psychol 2013, 122 (1): 45-50.

[17] Thayer RE, Newman JR, McClain TM: Self-regulation of mood: strategies for changing a bad mood, raising energy, and reducing tension. Journal of personality and social psychology 1994, 67 (5): 910-925.

[18] Daley A: Exercise and premenstrual symptomatology: a comprehensive review. Journal of women's health 2009, 18 (6): 895-899.

[19] Levesque J, Eugene F, Joanette Y, Paquette V, Mensour B, Beaudoin G, Leroux JM, Bourgouin P, Beauregard M: Neural circuitry underlying voluntary suppression of sadness. Biological psychiatry 2003, 53 (6): 502-510.

[20] Galdo Alvarez S, Lindin Novo M, Diaz Fernandez F: Naming faces: a multidisciplinary and integrated review. Psicothema 2009, 21 (4): 521-527.

[21] Chammat M, Foucher A, Nadel J, Dubal S: Reading sadness beyond human faces. Brain research 2010, 1348: 95-104.

[22] Dennis TA, Chen CC: Emotional face processing and attention performance in three domains: neurophysiological mechanisms and moderating effects of trait anxiety. International journal of psychophysiology: official journal of the International Organization of Psychophysiology 2007, 65 (1): 10-19.

[23] Nasr S, Esteky H: A study of N250 event-related brain potential during face and non-face detection tasks. Journal of vision 2009, 9 (5): 5, 1-14.

[24] Wijers AA, Van Besouw NJ, Mulder G: Selective attention to a facial feature with and without facial context: an ERP-study. International journal of psychophysiology: official journal of the International Organization of Psychophysiology 2002, 44 (1): 13-35.

[25] Streit M, Wolwer W, Brinkmeyer J, Ihl R, Gaebel W: EEGcorrelates of facial affect recognition and categorisation of blurred faces in schizophrenic patients and healthy volunteers. Schizophrenia research 2001, 49 (1-2): 145-155.

[26] Wynn JK, Lee J, Horan WP, Green MF: Using event related potentials to explore stages of facial affect recognition deficits in schizophrenia. Schizophrenia bulletin 2008, 34 (4): 679687.

[27] Streit M, Wolwer W, Brinkmeyer J, Ihl R, Gaebel W: Electrophysiological correlates of emotional and structural face processing in humans. Neurosci Lett 2000, 278 (1-2): 1316.

[28] Luo W, Feng W, He W, Wang NY, Luo YJ: Three stages of facial expression processing: ERP study with rapid serial visual presentation. NeuroImage 2010, 49 (2): 1857-1867.

[29] Balconi M, Pozzoli U: Event-related oscillations (ERO) and event-related potentials (ERP) in emotional face recognition. The International journal of neuroscience 2008, 118 (10): 1412-1424. 
[30] Carretie L, Martin-Loeches M, Hinojosa JA, Mercado F: Emotion and attention interaction studied through eventrelated potentials. Journal of cognitive neuroscience 2001, 13 (8): 1109-1128.

[31] Streit M, Ioannides AA, Liu L, Wolwer W, Dammers J, Gross J, Gaebel W, Muller-Gartner HW: Neurophysiological correlates of the recognition of facial expressions of emotion as revealed by magnetoencephalography. Brain research Cognitive brain research 1999, 7 (4): 481-491.

[32] Wolwer W, Brinkmeyer J, Stroth S, Streit M, Bechdolf A, Ruhrmann S, Wagner M, Gaebel W: Neurophysiological correlates of impaired facial affect recognition in individuals at risk for schizophrenia. Schizophrenia bulletin 2012, 38 (5): 1021-1029.

[33] Raichlen DA, Alexander GE: Adaptive Capacity: An Evolutionary Neuroscience Model Linking Exercise, Cognition, and Brain Health. Trends in neurosciences 2017, 40 (7): 408-421.

[34] Matta Mello Portugal E, Cevada T, Sobral Monteiro-Junior R, Teixeira Guimaraes T, da Cruz Rubini E, Lattari E, Blois C, Camaz Deslandes A: Neuroscience of exercise: from neurobiology mechanisms to mental health. Neuropsychobiology 2013, 68 (1): 1-14.

[35] Breiter HC, Etcoff NL, Whalen PJ, Kennedy WA, Rauch SL, Buckner RL, Strauss MM, Hyman SE, Rosen BR: Response and habituation of the human amygdala during visual processing of facial expression. Neuron 1996, 17 (5): 875-887.

[36] Steiner M, Macdougall M, Brown E: The premenstrual symptoms screening tool (PSST) for clinicians. Archives of women's mental health 2003, 6 (3): 203-209.

[37] Kerestes R, Labuschagne I, Croft RJ, O'Neill BV, Bhagwagar Z, Phan KL, Nathan PJ: Evidence for modulation of facial emotional processing bias during emotional expression decoding by serotonergic and noradrenergic antidepressants: an event-related potential (ERP) study. Psychopharmacology 2009, 202 (4): 621-634.

[38] Liu T, Pinheiro A, Zhao Z, Nestor PG, McCarley RW, Niznikiewicz MA: Emotional cues during simultaneous face and voice processing: electrophysiological insights. PloS one 2012, 7 (2): e31001.

[39] Fox KR: The influence of physical activity on mental wellbeing. Public Health Nutr 1999, 2 (3A): 411-418.

[40] Kramer AF, Erickson KI: Capitalizing on cortical plasticity: influence of physical activity on cognition and brain function. Trends in cognitive sciences 2007, 11 (8): 342-348.

[41] Bernstein EE, McNally RJ: Acute aerobic exercise helps overcome emotion regulation deficits. Cognition \& emotion 2017, 31 (4): 834-843.

[42] Crabbe JB, Smith JC, Dishman RK: Emotional \& electroencephalographic responses during affective picture viewing after exercise. Physiology \& behavior 2007, 90 (2-3): 394-404.

[43] Korzekwa MI, Steiner M: Premenstrual syndromes. Clinical obstetrics and gynecology 1997, 40 (3): 564-576.

[44] Kraemer GR, Kraemer RR: Premenstrual syndrome: diagnosis and treatment experiences. $J$ Womens Health 1998, 7 (7): 893907.
[45] Roy EJ, Wilson MA, Kelley DB: Estrogen-induced progestin receptors in the brain and pituitary of the South African clawed frog, Xenopus laevis. Neuroendocrinology 1986, 42 (1): 51-56.

[46] Huppertz C, Bartels M, Groen-Blokhuis MM, Dolan CV, de Moor MH, Abdellaoui A, van Beijsterveldt CE, Ehli EA, Hottenga JJ, Willemsen $\mathrm{G}$ et al: The dopaminergic reward system and leisure time exercise behavior: a candidate allele study. BioMed research international 2014, 2014: 591717.

[47] Tsuchiya N, Iwase M, Izumizaki M, Homma I: Dopaminergic modulation of exercise hyperpnoea via $D(2)$ receptors in mice. Experimental physiology 2012, 97 (2): 228-238.

[48] Baj G, D'Alessandro V, Musazzi L, Mallei A, Sartori CR, Sciancalepore M, Tardito D, Langone F, Popoli M, Tongiorgi E: Physical exercise and antidepressants enhance BDNF targeting in hippocampal CA3 dendrites: further evidence of a spatial code for BDNF splice variants. Neuropsychopharmacology 2012, 37 (7): 1600-1611.

[49] Ma Q: Beneficial effects of moderate voluntary physical exercise and its biological mechanisms on brain health. Neuroscience bulletin 2008, 24 (4): 265-270.

[50] Moon HY, Kim SH, Yang YR, Song P, Yu HS, Park HG, Hwang O, Lee-Kwon W, Seo JK, Hwang D et al: Macrophage migration inhibitory factor mediates the antidepressant actions of voluntary exercise. Proceedings of the National Academy of Sciences of the United States of America 2012, 109 (32): 13094-13099.

[51] Wu G, Feder A, Cohen H, Kim JJ, Calderon S, Charney DS, Mathe AA: Understanding resilience. Frontiers in behavioral neuroscience 2013, 7: 10.

[52] Baratta MV, Rozeske RR, Maier SF: Understanding stress resilience. Frontiers in behavioral neuroscience 2013, 7: 158.

[53] Gross JJT, R. A.: Emotion regulation: Conceptual foundations. In JJ Gross (Ed), Handbook of emotion regulation (pp 3-26) New York: The Guilford Press 2007.

[54] Lane AM, Beedie CJ, Jones MV, Uphill M, Devonport TJ: The BASES expert statement on emotion regulation in sport. Journal of sports sciences 2012, 30 (11): 1189-1195.

[55] Dietrich A: Transient hypofrontality as a mechanism for the psychological effects of exercise. Psychiatry Res 2006, 145 (1): 79-83.

[56] Lee YY, Hsieh S: Classifying different emotional states by means of EEG-based functional connectivity patterns. PloS one 2014, 9 (4): e95415.

[57] Kalisch R, Wiech K, Herrmann K, Dolan RJ: Neural correlates of self-distraction from anxiety and a process model of cognitive emotion regulation. Journal of cognitive neuroscience 2006, 18 (8): 1266-1276.

[58] Berkman ET, Lieberman MD: Approaching the bad and avoiding the good: lateral prefrontal cortical asymmetry distinguishes between action and valence. Journal of cognitive neuroscience 2010, 22 (9): 1970-1979.

[59] Barnes RT, Coombes SA, Armstrong NB, Higgins TJ, Janelle $\mathrm{CM}$ : Evaluating attentional and affective changes following an acute exercise bout using a modified dot-probe protocol. Journal of sports sciences 2010, 28 (10): 1065-1076. 
[60] Dixon ML: Cognitive control, emotional value, and the lateral prefrontal cortex. Frontiers in psychology 2015, 6: 758.

[61] Kouneiher F, Charron S, Koechlin E: Motivation and cognitive control in the human prefrontal cortex. Nature neuroscience 2009, 12 (7): 939-945.

[62] Morawetz C, Bode S, Baudewig J, Heekeren HR: Effective amygdala-prefrontal connectivity predicts individual differences in successful emotion regulation. Social cognitive and affective neuroscience 2017, 12 (4): 569-585.

[63] Ochsner KN, Bunge SA, Gross JJ, Gabrieli JD: Rethinking feelings: an FMRI study of the cognitive regulation of emotion. Journal of cognitive neuroscience 2002, 14 (8): 1215-1229.

[64] Baxter LR: Brain imaging as a tool in establishing a theory of brain pathology in obsessive compulsive disorder. The Journal of clinical psychiatry 1990, 51 Suppl: 22-25; discussion 26.
[65] LeDoux J, 1996. The Emotional Brain. Touchstone, New York.

[66] Mayberg HS: Limbic-cortical dysregulation: a proposed model of depression. The Journal of neuropsychiatry and clinical neurosciences 1997, 9 (3): 471-481.

[67] Dishman RK: Brain monoamines, exercise, and behavioral stress: animal models. Medicine and science in sports and exercise 1997, 29 (1): 63-74.

[68] Salmon P: Effects of physical exercise on anxiety, depression, and sensitivity to stress: a unifying theory. Clinical psychology review 2001, 21 (1): 33-61. 